\title{
Radiobiological rationale for Stereotactic Hypofractionated Radiosurgery (SHRS) Part I. LQED 2 or BED formalism
}

\author{
Bogusław Maciejewski ${ }^{1}$, Sławomir Blamek ${ }^{1}$, Krzysztof Składowski², Rafał Suwiński ${ }^{3}$ \\ Leszek Miszczyk ${ }^{1}$, Krzysztof Ślosarek ${ }^{4}$, Marcin Miszczyk ${ }^{4}$
}

In conventional radiotherapy, 5R's mechanisms influence tumour cell kill, but in SHRS they do not sufficiently explain the biology of large doses. Indirect cell death is also induced by endothelial damage, stem cell death and antitumour immunity are also activated by a single dose $\geq 12-15 \mathrm{~Gy}$. These three processes defined as extra 3R's are characterizers in details. Despite some controversies, LQED formalism seems not quite adequate for SHRS. Experimental and a few clinical studies suggest BED formalism as much more useful. Both formalisms are compared and discussed. Clinical reports show a monotonical increase in Tumour Cure Probability (TCP) with higher BED doses. The advantage of SHRS results in significant shortening overall treatment time and in delivery of the BED doses higher than $100 \mathrm{~Gy}$, producing an increase in the TCP, likely unachievable by conventional dose fractionation.

NOWOTWORY J Oncol 2018; 68, 1: 8-14

Key words: SHRS radiobiology, indirect extra 3R's, BED in favour of LQED

Stereotactic Hypofractionated Radiosurgery (SHRS) with high single dose (i.e. 10-30 Gy) or a few large fractions has come to the market as an attractive and highly effective method alternative to conventional or altered fractionation regimes. It is used not only to irradiate brain tumours, but it has been also applied to extracranial tumours [1-4]. The results, mainly empirical, show this method to be undoubtedly highly effective and well tolerated (Fig. 1). Although SHRS is more and more widely applied in practice, it is in some way hampered by the still debatable background of its radiobiology.

\section{Radiobiological rationale for SHRS - 5R's or 8R's}

Five radiobiological processes ( $5 \mathrm{R}$ 's) which regulate cellular malignant and normal tissue response to radiation are well recognized. They work in opposite directions, depending on tumour type and dose fractionation schedules. As a response to conventional fractionation redistribution and reoxygenation increase cell killing, whereas on the contrary, repair and repopulation improve cell survival.

Considering SHRS, important question arises whether the $5 R$ 's are the only mechanisms which decide on final cell kill effect of a high single or a few large fractions. Clinical studies on SHRS of various primary or metastatic tumour sites have documented local tumour control rates significantly superior to those achieved by conventional fractionation. Therefore, antitumour efficacy of SHRS is greater than would be predicted by classic survival curves. Brown et al. [5] suggest that in case of SHRS, there are some biological challengers to the $5 R^{\prime}$ s.

\footnotetext{
${ }^{1}$ Department of Radiotherapy, Maria Skłodowska-Curie Memorial Cancer Centre and Institute of Oncology, Branch in Gliwice, Poland

${ }^{2} 1^{\text {st }}$ Clinic of Radiotherapy and Chemotherapy, Maria Skłodowska-Curie Memorial Cancer Centre and Institute of Oncology, Branch in Gliwice, Poland

${ }^{3} 3^{\text {rd }}$ Clinic of Radiotherapy and Chemotherapy, Maria Skłodowska-Curie Memorial Cancer Centre and Institute of Oncology, Branch in Gliwice, Poland

${ }^{4}$ Department Radiotherapy Treatment Planning, Maria Skłodowska-Curie Memorial Cancer Centre and Institute of Oncology, Branch in Gliwice, Poland
} 


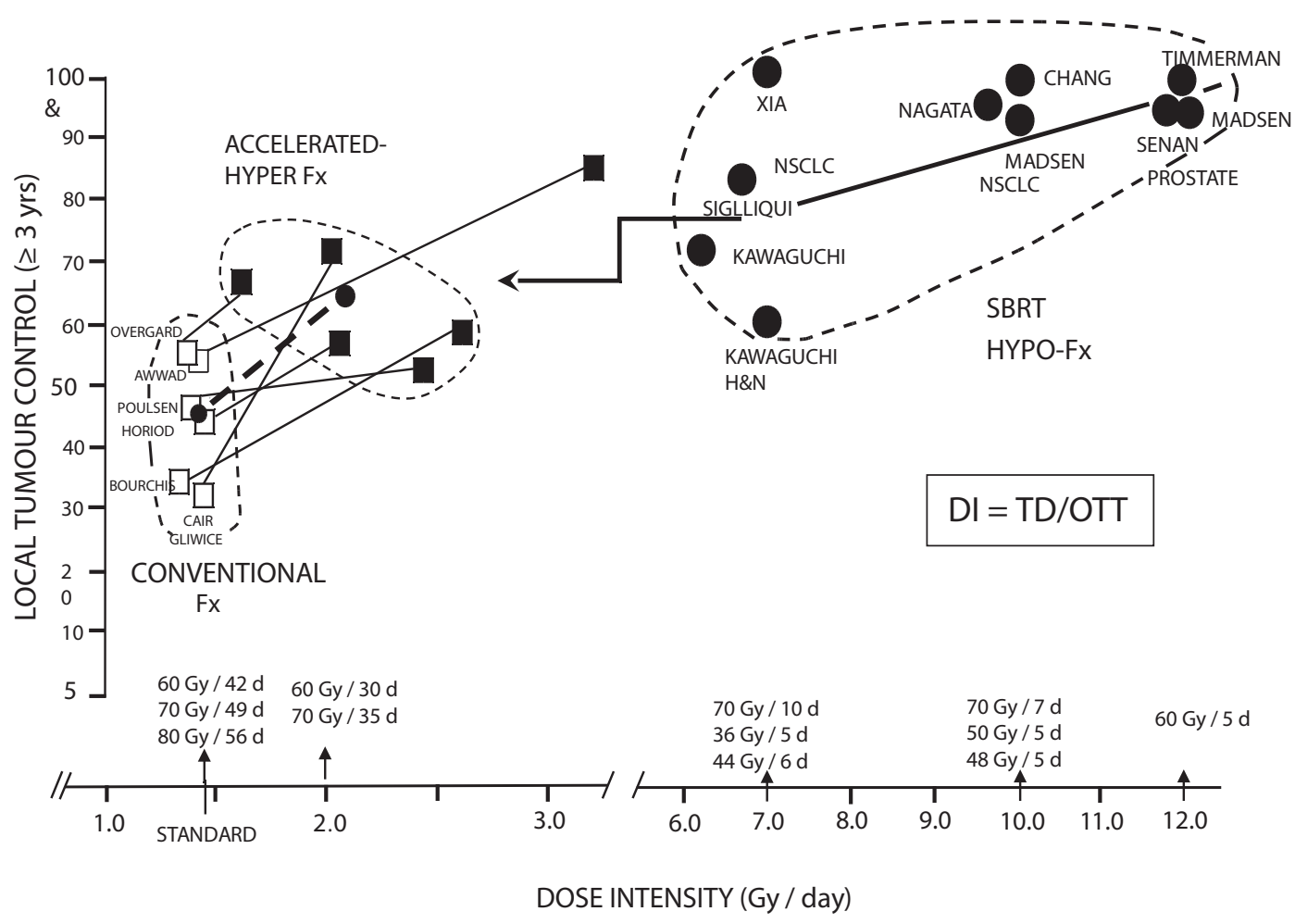

Figure 1. Local tumor control (TCP) for conventional altered and stereotactic hypofractionated radiotherapy related to Dose Intensity (number of Gray per day)

Experimental studies $[6,7]$ have shown that the radiosensitivity of tumours to dose fractions of $10 \mathrm{~Gy}$ or more is governed by the sensitivity of the tumour endothelial cells to apoptosis. Park et al. [8] and Song et al. [9] postulate that vascular damage induced by high doses indirectly leads to tumour cell death. Another study [10] reports enhancement of antitumour immunity by doses higher than $10 \mathrm{~Gy}$. Moreover, tumour hypoxia may also play an important role.

The cell survival model in Figure 2 illustrates concepts proposed by Park [8], Brown [5] and Song [9]. The combination of three survival curves suggests that the cellular response to a single dose of $0-5 \mathrm{~Gy}$ is likely dominated by oxic cells, whereas in the range of 5-12 Gy it is rather dominated by hypoxic cells. Above $12 \mathrm{~Gy}$, the overall survival curve sharply straightens downwards, which may likely correspond with indirect cell death due to vascular endothelial cell damage, and enhanced antitumour immunity. These mechanisms called an extra 3R's probably increase the antitumour efficacy of high SHRS doses, which cannot be simply explained by the classic $5^{\prime}$ Rs.

Kocher et al. simulated a vascular effect after single dose of 20 Gy [11]. Park et al. summarized 43 studies on the radiation-induced vascular damage either in human tumours or in their xenografts [8]. Both authors concluded that the therapeutic effect of SHRS is likely dominantly influenced by vascular effects. Large SHRS doses cause severe vascular damage and nutritional deprivation of the intratumour microenvironment which induce indirect tumour cell death. Vascular effects mainly occur after doses of at least $12 \mathrm{~Gy}$ and higher (Fig. 2 and Fig. 3) and they do not necessarily accompany direct effects of cell kill but are usually delayed and occur at random time between the onset of irradiation and about 1 year thereafter. Dose-equivalent vascular effect was counted at the level of about $19-23 \%$ of total dose.

Clinical results with SHRS, especially for early-stage I non-small cell lung cancer $[5,12]$ are impressive and show high local tumour control, superior to that achieved by conventional fractionation. Although many different single or a few large fractions are used nowadays in practice, the importance of the extra 3R's is still only qualitatively estimated. Its quantitation is not simple, and not yet available. Brown et al. [5] conclude that more experimental evidence is needed to prove an important role of the extra 3R's in increasing tumour sensitivity and its response to high dose fractions.

It is generally well recognized that hypoxic cells are more radioresistant than oxic ones and likely tumour hypoxia is a detrimental factor for SHRS. Within the hierarchy of tumour cells, there is a selected subpopulation which is capable of self-renewal, and these are called stem cells, being more radioresistant than others $[13,14]$. There is still a belief in rather than evidence of higher radioresistance of the stem cells, since their characteristics have not yet been quantified. 


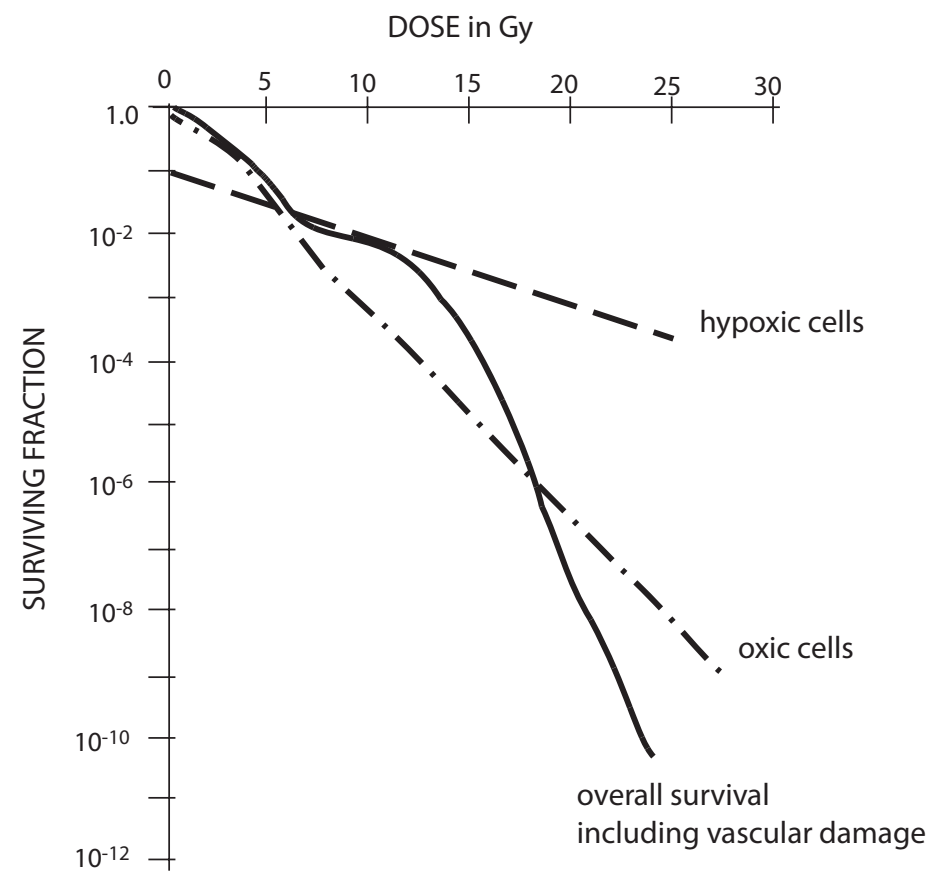

Figure 2. Scheme of cell survival after large single dose including direct and indirect cell death (vascular damage, stem cell death) (according to Song et al. [9] and Brown et al. [5])

Nevertheless, their response to radiation or other cytotoxic agents share with other factors an impact on SHRS clinical outcome.

Ch'ang et al. [15] have shown that at very high doses of more than 17-18 Gy, a radiation threshold is crossed and stem cells die due to programmed cell death. It seems reasonable to assume that such a threshold may likely exist in human tumours. Kirkpatrick et al. [16] have simulated cell survival curves using a LQ model with $\alpha=0.3 \mathrm{~Gy}^{-1}$ and $\beta=0.03 \mathrm{~Gy}^{-2}$, including a threshold dose for vascular damage. In the present paper, their model is supplemented by adding an anticipated effect of antitumour immunity and stem cell death (Fig. 3). High doses evoke immune reactions, which among others, induce an increase number of cytotoxic T-cells which help to eradicate the response of the primary tumour or distant metastases [10]. Kirpatrick et al. [16] argue that the LQ model underestimates radiation cell killing at elevated doses because the

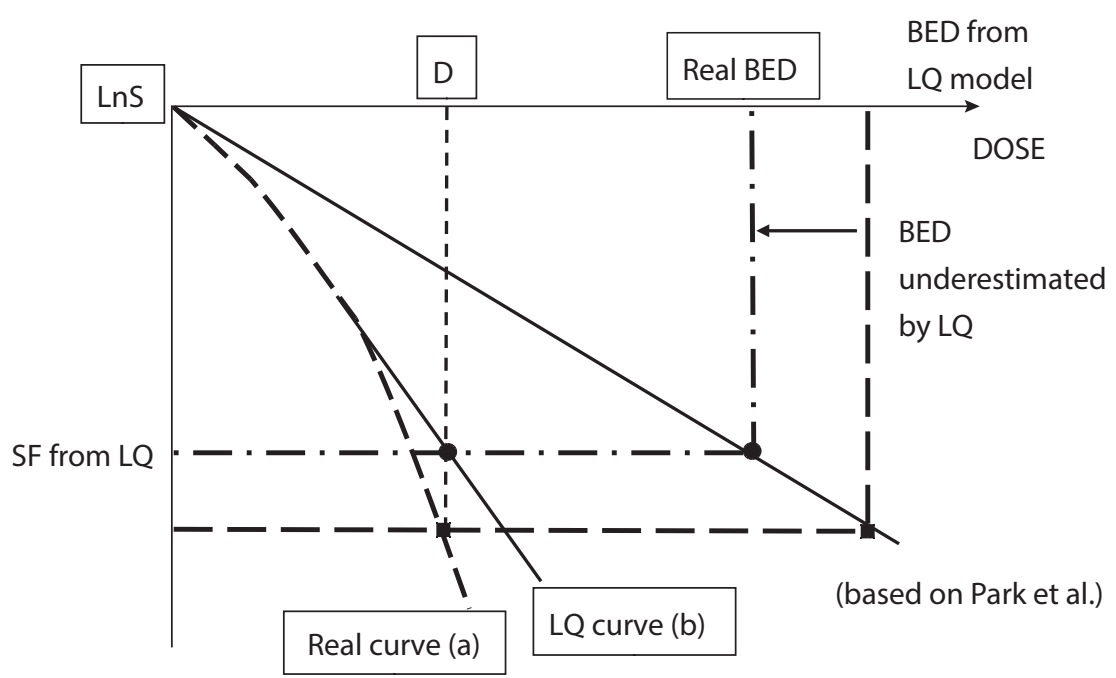

Above 13-15 Gy survival curve becomes largely linear leading to lower survival fraction than that predicted from lq formalism

Figure 3. Tumour cell survival curves representing various direct and indirect radiobiological effects (modified from Kirkpatrick et al. [16]) 
model predicts a continuous downward bending survival curve (due to $\beta d^{2}$ term), whereas dose-response data are likely linear at doses above $12 \mathrm{~Gy}$ (Fig. 4). Finally, the authors suggest that the LQ model may be inappropriate to design SHRS fraction doses, arguing that clinical response to radiotherapy does not only reflect $\alpha$ and $\beta$ effects.

\section{LQED ${ }_{2}$ and BED formalisms}

The Mechanistic Linear-Quadratic (LQ) model of cell killing is commonly used to compare biologically doses of two or more regimes of conventional radiotherapy by calculation of equivalent doses to that if given in 2 Gy fractions. This formalism was defined by Fowler [17] as LQED 2 (term NTD is also used):

$$
\operatorname{LQED}_{2}=\mathrm{D}_{\mathrm{i}}\left(\alpha / \beta+\mathrm{d}_{\mathrm{i}}\right) /(\alpha / \beta+2.0 \mathrm{~Gy})
$$

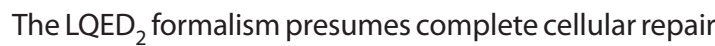
between fractions and neglects the role of treatment time (cellular repopulation). Nevertheless, this formalism provides a good fit for the majority of conventional external radiotherapy.

An alternative approach is based on the assumption of a purely theoretical regimen with a total dose delivered in an infinitive number of infinitively small fraction doses $\left(d_{i}\right)$ decreasing to $d_{i}=0$, with complete repair of sublethal damage between fractions. Transforming Eq. 1 by setting a $2.0 \mathrm{~Gy}$, model expresses the biological total dose, defined by Fowler [17] as the Biologically Effective Dose (BED) is:

$$
\mathrm{BED}=\mathrm{D}_{\mathrm{i}}\left(1+\frac{\mathrm{d}_{\mathrm{i}}}{\alpha / \beta}\right)
$$

where $D_{i}$ and $d_{i}$ are total and fraction dose respectively. However, both the LQED 2 and BED formalisms ignore effects of inhomogeneous dose distribution within irradiated volume.

Although the physical DVH illustrates the proportions of the volume receiving dose above or below assumed level, it does not illustrate its localization in the anatomical space of high-or low-dose regions. "Hot spots" within tumour volume are acceptable or even expected, whereas within critical normal tissues these can be disastrous, especially in organs (e.g. spinal cord) with serial structure, even if they are isolated and small. For such structures failure of any small element is critical. In contrast, "cold spots" within the tumour volume may lead to local failure, or at least to a lesser or greater decrease in the predicted probability of local tumour control.

According to Brenner [18], the LQ model seems reasonably predictive for a response to dose per fraction in the range of 2 to $18 \mathrm{~Gy}$. Although the model might be progressively less accurate above $15 \mathrm{~Gy}$, it could be useful in clinical studies within acceptable frames. At very high doses (> 17-18 Gy), the threshold is crossed, and tumour stem cells die as a result of programmed cell death, likewise of vascular damage leading to necrotic death in the majority of tumour cells, 2 to 3 days after irradiation or a bit later.

Despite some suggestions that $\mathrm{LQED}_{2}$ cannot logically work for SHRS (Fig. 4), because $60 \mathrm{~Gy}$ in 30 fractions is absolutely not equivalent to $60 \mathrm{~Gy}$ in 3 fractions. It is obvious that $\alpha$ and $\beta$ factors in the LQED 2 model do not quantitively express all biological mechanisms induced by large single or fractionated doses. The $L Q E D_{2}$ model likely underestimates

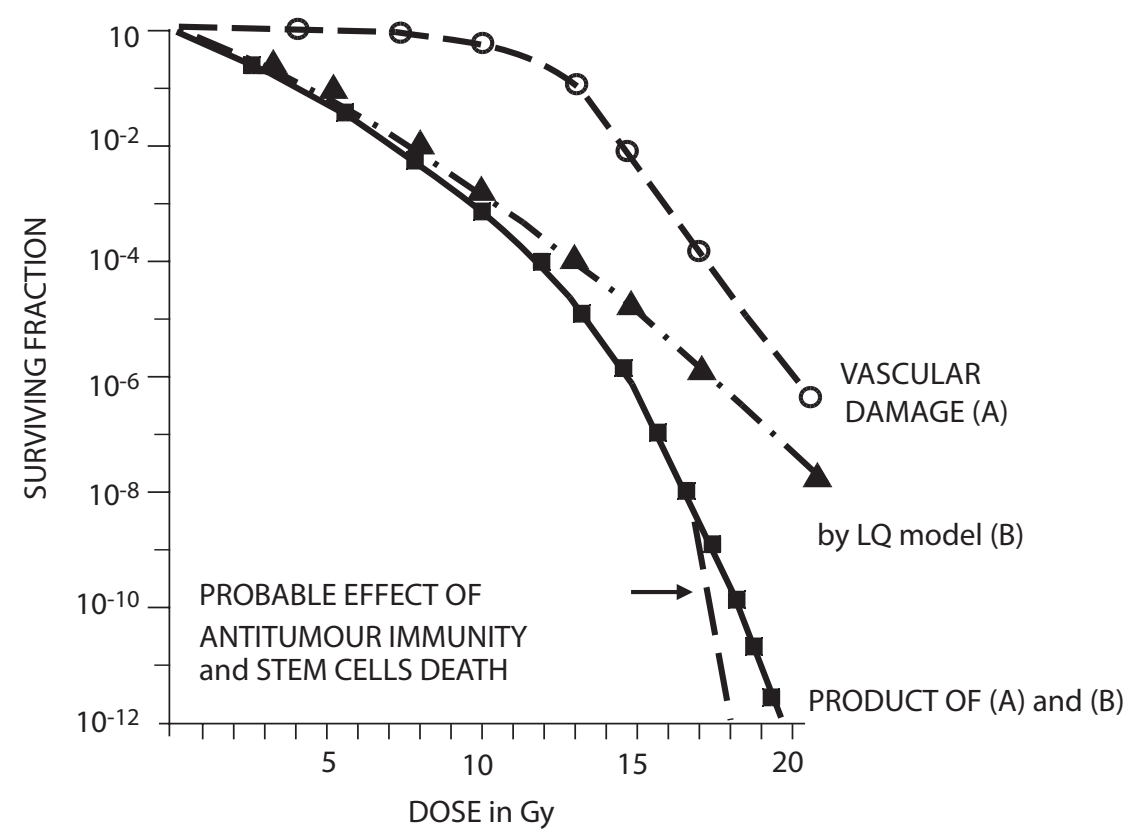

Figure 4. Underestimation of the BED calculated using the LQ model compared with the BED determined from empirical survival curve (from Park et al. [8]), SF — surviving fraction 
radiation cell killing at higher doses (Fig. 4). The cell survival curve above 3-15 Gy becomes largely linear, leading to considerably lower surviving fraction than that predicted by the LQED 2 model. However, until now, there is a lack of quantitative data regarding the impact of indirect features on the shape and steepness of the tumour cell survival curve.

\section{Is BED formalism favourable for SHRS?}

Aside from $\alpha$ and $\beta$ factors, there is another "handy" and attractive radiobiological parameter, defined as $\mathrm{e}_{0}$, which characterizes the exponential part of the cell survival curve. In vitro and some clinical studies convincingly document the $\mathrm{D}_{0}$ of about 3.0 Gy for 2.0 Gy fraction regimens. The $\mathrm{e}_{0}$ decreases the surviving fraction (SF) to $\mathrm{e}^{-1}$ (this means to 0.37 of the initial number of tumour cells). However, it is not easy to apply ${ }_{e} D_{0}$ in practice, and the ${ }_{e} D_{10}$ parameter is more practical (Appendix 1) because it results in the decreases of cell surviving fraction by one decade of cells (e. q. from $10^{9}$ to $10^{8}$ ). For the majority of solid tumours, $\mathrm{D}_{10}$ is about $7 \mathrm{~Gy}$ $\left({ }_{e} D_{10}=2.3 \times{ }_{e} D_{0}\right)$. If, for example, TCP of $90 \%$ is planned for tumour with $10^{10}$ cells, then it requires a decrease of the surviving fraction to $10^{-11}\left(\mathrm{TCP}_{90}=\mathrm{e}^{-}\left(10^{10} \cdot 10^{-11)}=\mathrm{e}^{-0.1}\right)\right.$ and the respective total dose in $2.0 \mathrm{~Gy}$ fractions would be $11 \times$ $7 \mathrm{~Gy}=77 \mathrm{~Gy}$. However, such an estimation does not work for SHRS because the $D_{10}$ of $7 \mathrm{~Gy}$ underestimated cell kill effect.

Based on the report of 12 NSCLC cell lines from the $\mathrm{NCl}$, Park et al. determined mean values for $\alpha=0.33 \mathrm{~Gy}^{-1}$, $e^{D_{0}}=1.23-1.65 \mathrm{~Gy}$ and $D_{q}=1.8 \mathrm{~Gy}$, parameters which describe the cell survival curve for a large fraction regimen with much lower $\mathrm{e}_{0}$ [8]. Using that $\mathrm{e}_{0}$ value, we calculate a respective $\mathrm{e}_{10}$ of 2.8-3.8 Gy. Thus, $\mathrm{D}_{10}$ for large fractions seems to about 2-times lower than that for $2 \mathrm{~Gy}$ regimens, and more effective because $7 \mathrm{~Gy}$ in one fraction of SHRS would reduce cell survival by two decades, compared with one decade of cells reduced by $7 \mathrm{~Gy}$ given in 3 fractions of conventional regimen. This proves that the $\mathrm{LQED}_{2}$ is not really representative for large fractions because it lowers its real biological power.

The majority of large fraction regimens have been designed empirically. Park et al. listed ten various large fraction regimens which were used to irradiate patients with early stage I of NSCLC [8]. It is difficult to explain why some authors use single dose of 10-20 Gy, whereas others prefer 20-34 Gy. We used different total physical doses given as a single or 3-4 fractions to estimate respective $B D_{10}$, using $\alpha / \beta=10$ Gy values. Figure 5 shows that a choice of SHRS regimes is not incidental since respective $\mathrm{BED}_{10}$ curves for single, 3 and 4 fractions give a good linear fit to the BED doses.

The BED formalism quite well fits a correlation between TCP and the BED values. To demonstrate this relationship, we used data from Fowler et al. for intermediate risk prostate cancer, to recalculate physical doses as $\mathrm{BED}_{2}$ [19]. Figure 6 shows the BNED (Biochemical No Evidence of Disease) original curve representing a conventional fractionation dose of $72 \mathrm{~Gy}$ in 2 fractions $\left(\mathrm{BED}_{1.5}=167 \mathrm{~Gy}_{1.5}\right.$ ) resulting in BNED of $69 \%$. Instead of increasing the conventionally fractionated

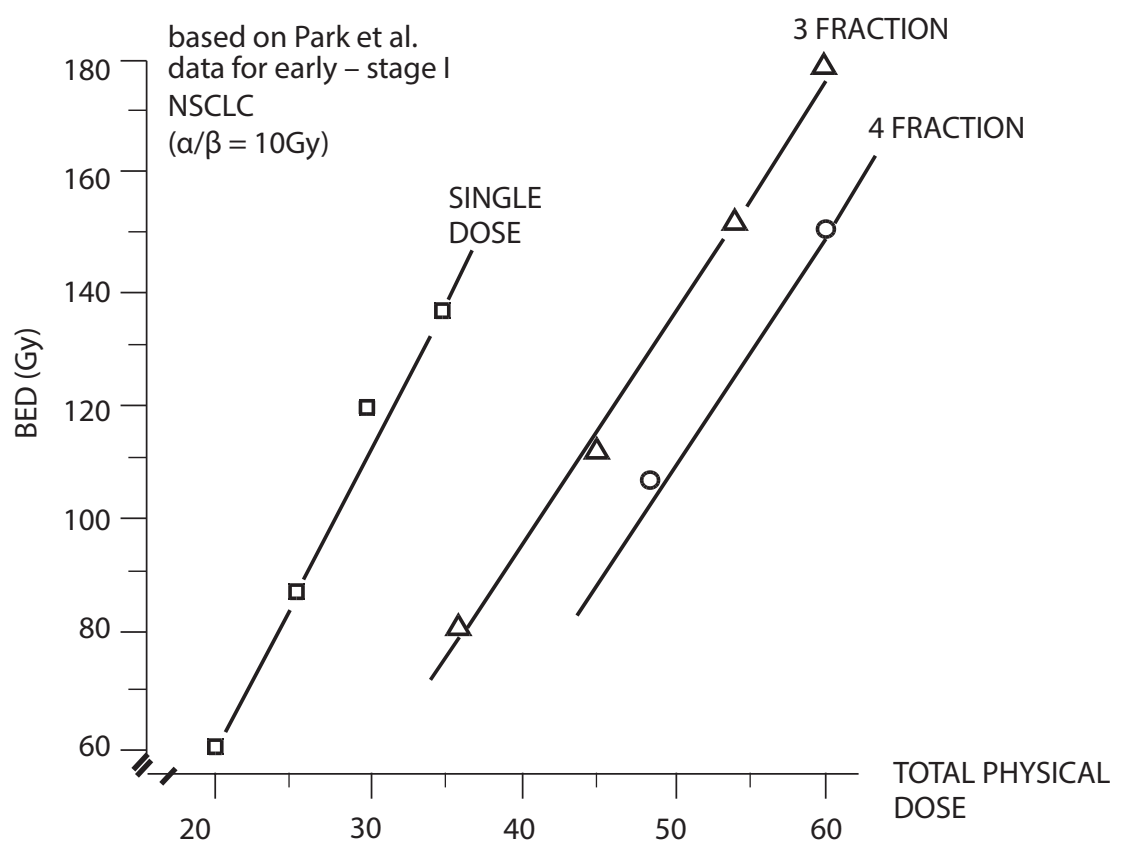

Figure 5. Relation between BED and single and 3-4 large fractions of physical dose estimated from Part et al. [8] data of SHRS for early-stage NSCLC 


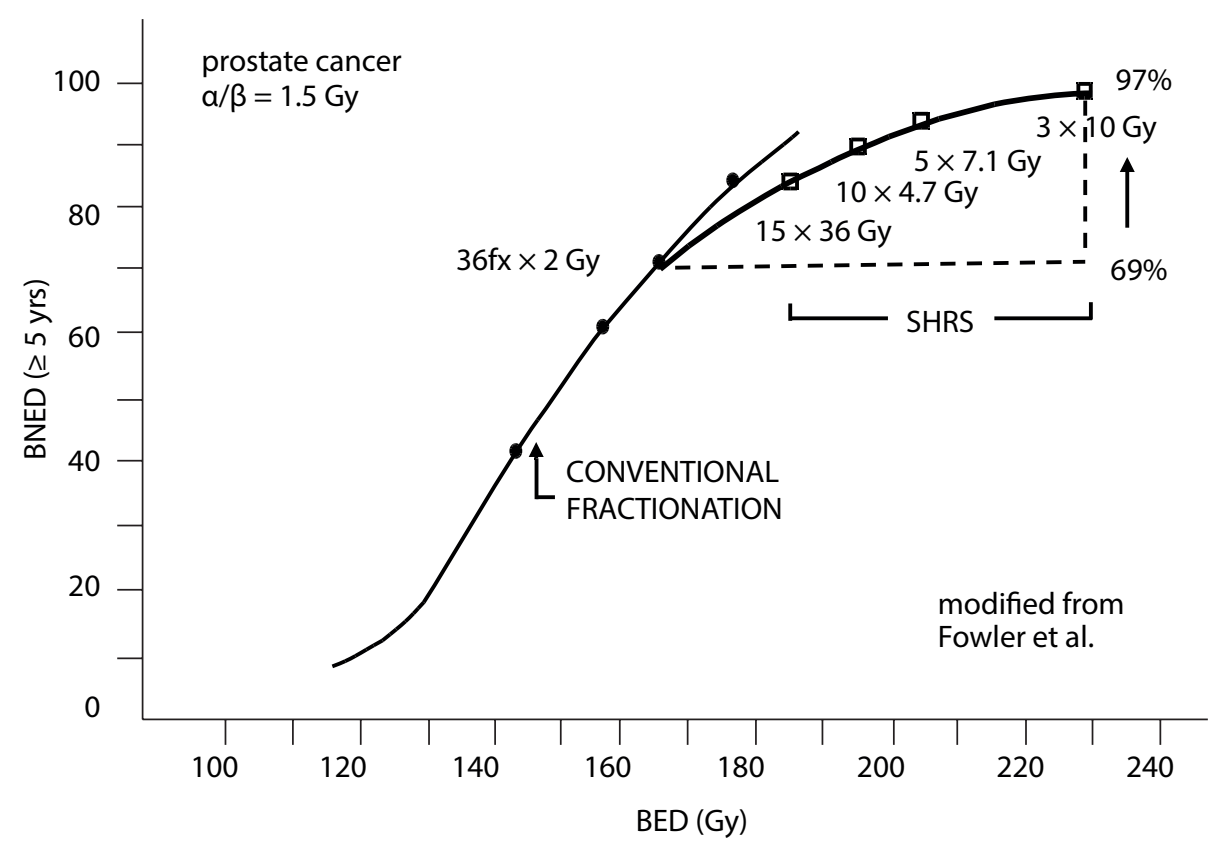

Figure 6. BNED for prostate cancer with intermediate risk as a function of the BED of conventional fractionation and simulation of SHRS regimes (based on Fowler et al. [19] data)

total dose, large fraction regimens, from $15 \times 3.6 \mathrm{~Gy}$ to $3 \times$ $10 \mathrm{~Gy}$ reflect an increasing $\mathrm{BED}_{2}$ from $167 \mathrm{~Gy}_{1.5}$ to $230 \mathrm{~Gy}_{1.5}$ which yields a $28 \%$ increase in BNED. Moreover, an important advantage of SHRS regimens is significant shortening of overall treatment time from 50 days to even 3-5 days.

The BED formalism as a version of the LQ model seems reliable for the design and comparison of various SHRS protocols even for fraction doses higher than $10 \mathrm{~Gy}$. It might be naive to believe that the clinical response to SHRS depends only on $\alpha$ and $\beta$ effects. It is much more reasonable to accept that additional indirect 3R's also participate in the response to SHRS, but these need to be validated experimentally and clinically. Clinical results show that TCP monotonically gets higher with an increase in BED, and the clinical benefit of SHRS is noted due to delivery of BED doses higher than $100 \mathrm{~Gy}$, which is unachievable by conventional dose fractionation.

\section{Conclusions}

High local efficacy (high TCP) of Stereotactic Hypofractionated Radiosurgery depends on the direct radiation cell kill (5R's) and additionally on indirect vascular damage, stem cells programmed death and antitumour immunity (extra 3R's) which can be counted as about $19-23 \%$ of total dose. Among LQ formalisms, the BED correlates with SHRS efficacy better than $\mathrm{LQED}_{2}$, because its values monotonically increase when SHRS single or fractionated doses get higher. High clinical efficacy of SHRS can be achieved due to BED delivery of doses higher than $100 \mathrm{~Gy}$, which is unachievable by conventional dose fractionation.

\section{Conflict of interest: none declared}

prof. Bogusław Maciejewski, MD, PhD

Maria Skłodowska-Curie Memorial Cancer Centre and Institute of Oncology

Deptartment of Radiotherapy

ul. Wybrzeże Armii Krajowej 15

44-101 Gliwice, Poland

e-mail:boguslaw.maciejewski@io.gliwice.pl

Received: 4 Apr 2018

Accepted: 10 Apr 2018

\section{References}

1. Ford E, Dieterich S. Safey consideration in stereotactic body radiation therapy. Semin Radiat Oncol 2017; 27: 190-196.

2. Timmerman R, Papiez L. McGarry R et al. Extracranial stereotactic radioablation: results of a phase I study in medically inoperable stage I non-small cell lung cancer. Chest 2003; 124: 1946-1955.

3. Hara R, Itami J, Kondo T et al. Clinical outcomes of single-fraction stereotactic radiotherapy of lung tumours. Cancer 2006; 106: 1347-1352.

4. Blomgren $\mathrm{H}, \mathrm{Lax}$ I, Naslund I et al. Stereotactic high dose fractionation radiation therapy of extracranial tumours using an accelerator. Clinical experience of the first thirty-one patients. Acta Oncol 1995; 34: 861-870.

5. Brown JM, Carlson DJ, Brenner DJ. The tumour radiobiology of SRS and SBRT: are more than the 5Rs involved? Int J Radiat Oncol Biol Phys 2014; 88: 254-262.

6. Garcia-Barros M, Paris F, Cordon-Cardo $C$ et al. Tumour response to radiotherapy regulated by endothelial cell apoptosis. Science 2003; 300: 1155-1159.

7. Fuks Z, Kolesnick R. Engaging the vascular component of the tumour response. Cancer Cell 2005; 8: 89-91.

8. Park HJ, Griffin RJ, Hui S et al. Radiation-induced vascular damage in tumours: implication of vascular damage in ablative hypofractionated radiotherapy (SBRT and SRS). Radiat Res 2012; 177: 311-327.

9. Song CW, Cho LC, Yuan J et al. Radiobiology of stereotactic body radiation therapy/stereotactic radiosurgery and the linear-quadratic model. Int J Radiat Oncol Biol Phys 2013; 87: 18-19. 
10. Lee $Y$, Auh $S L$, Wang $Y$ et al. Therapeutic effect of ablative radiation on local tumour require CD8+ T cells: changing strategies for cancer treatment. Blood 2009; 114: 589-595.

11. Kocher M. Treuer $\mathrm{H}$, Voges J et al. Computer simulation of cytotoxic and vascular effects of radiosurgery in solid and necrotic brain metastases. Radiother Oncol 2000; 54: 149-156.

12. Ivengar P, Timmerman RD. Stereotactic ablative radiotherapy for non-small cell lung cancer: rationale and outcomes. J Nat/ Compr Cancer Netw 2012; 10: 1514-1520.

13. Uchida N, Buck DW, He D et al. Direct isolation of human central nervous system stem cells. Proc Nat/ Acad SciUSA 2000; 97: 14720-14725.

14. Singh SK, Clarke ID, Terasaki $M$ et al. Identification of cancer stem cell in human brain tumours. Cancer Res 2003; 63: 5821-5828.
15. Ch'ang HJ, Maj JG, Paris F et al. ATM regulates target switching to escalating doses of radiation in the intestines. Nat Med 2005; 11: 484-490.

16. Kirkpatrick JP, Mayer JJ, Marks LB. The linear-quadratic model is inappropriate to model high dose per fraction effects in radiosurgery. Semin Radiat Oncol 2008; 18: 240-243.

17. Fowler JF. The linear-quadratic formula and progress in fractionated radiotherapy. Br J Radiol 1989; 62: 679-694.

18. Brenner DJ.The linear-quadratic model is an appropriate methodology for determining isoeffective doses at large doses per fraction. Semin Radiat Oncol 2008; 18: 234-239.

19. Fowler JF, Ritter MA, Chappell RJ et al. What hypofractionated protocol should be tested for prostate cancer? Int J Radiat Oncol Biol Phys 2003; 56: 1093-1104.

\section{Appendix 1}

The slope of the exponential part of cell survival curve is described by effective $D_{0}\left({ }_{e} D_{0}\right)$.

For dose $D$ dose equals $1_{e} D_{0}$ :

$\mathrm{SF}=\mathrm{e}^{-(1 \mathrm{eD} 0 / 1 \mathrm{eD} 0)}$

$=\mathrm{e}^{-1}$

$=0.37$

Therefore, one $\mathrm{D}_{0}$ reduces cell survival (SF) to 0.37

The ${ }_{e} D_{0}$ is not very useful to estimate $S F$ for a multifraction regimen, therefore

if dose $D$ equals $2.3 \mathrm{D}_{0}$ then:

$\mathrm{SF}=\mathrm{e}^{-(2.3 \mathrm{eD} 0 / 1 \mathrm{eD})}$

(b)

$=\mathrm{e}^{-2.3}$

$=0.1$

Thus $2.3 \times{ }_{e} D_{0}$ reduces cell survival by one decade of cells (i.e. from $10^{-2}$ to $10^{-1}$ ) and it is defined as $\mathrm{D}_{10}$ and $\mathrm{e}^{\mathrm{D}_{10}}=2.3 \times \mathrm{e}_{\mathrm{e}}$

In radiotherapy $\mathrm{D}_{0}$ for $2.0 \mathrm{~Gy}$ fraction regimen is on average $\sim 3.0 \mathrm{~Gy}$. From that

$\mathrm{e}^{\mathrm{D}_{10(2.0 \text { Gy fractions })}}=2.3 \times 3 \mathrm{~Gy}=6.9 \mathrm{~Gy} \sim 7 \mathrm{~Gy}$ 